\title{
A Probe-Shaped Sensor With FBG and Fiber-Tip Bubble for Pressure and Temperature Sensing
}

\author{
Bonan LIU ${ }^{1,2}$, Junxian LUO ${ }^{1,2}$, Shen LIU ${ }^{1,2 *}$, Yanping $\mathrm{CHEN}^{1,2}$, \\ Bo HUANG ${ }^{1,2}$, Changrui LIAO ${ }^{1,2}$, and Yiping WANG ${ }^{1,2}$
}

\author{
${ }^{1}$ Shenzhen Key Laboratory of Photonic Devices and Sensing Systems for Internet of Things, College of Physics and \\ Optoelectronic Engineering, Shenzhen University, Shenzhen 518060, China \\ ${ }^{2}$ Guangdong and Hong Kong Joint Research Centre for Optical Fibre Sensors, Shenzhen University, Shenzhen 518060, \\ China \\ *Corresponding author: Shen LIUＥ-mail: shenliu@szu.edu.cn
}

\begin{abstract}
A probe-shaped sensor for simultaneous temperature and pressure measurement was reported in this article. The effective length of the sensor was $\sim 2 \mathrm{~mm}$, consisting of a fiber Bragg grating (FBG) and a Fabry-Perot interferometer (FPI) with a nano silica diaphragm. The response sensitivities of the sensor for pressure and temperature were measured as $-0.98 \mathrm{~nm} / \mathrm{MPa}$ and $11.10 \mathrm{pm} /{ }^{\circ} \mathrm{C}$, respectively. This sensor had an extremely low cross-sensitivity between pressure and temperature, which provided a significant potential in dual-parameter sensing.
\end{abstract}

Keywords: Fabry-Perot interferometer; fiber Bragg grating; fiber optics sensors

Citation: Bonan LIU, Junxian LUO, Shen LIU, Yanping CHEN, Bo HUANG, Changrui LIAO, et al., "A Probe-Shaped Sensor With FBG And Fiber-Tip Bubble for Pressure and Temperature Sensing," Photonic Sensors, 2021, 11(4): 411-417.

\section{Introduction}

In the past two decades, optical fiber information technology has achieved a huge development. Optical-fiber-based sensors have attracted considerable attention due to their outstanding advantages of durability, flexibility, biocompatibility, high sensitivity, and electromagnetic interference immunity. For the indisputable superiorities, the optical fiber sensor is widely applied in many fields, such as industrial production, medical application, environmental protection, and monitoring quality of construction. A variety of optical fiber sensors have been fabricated to gather information of multifarious physical and chemical parameters, such as temperature [1], pressure [2], curvature [3, 4], humidity [5], and refractive index (RI) [6]. In order to fabricate these sensors, various kinds of fibers are adopted, such as photonic crystal fiber (PCF) [7, 8], polarization-maintaining fiber [9, 10], twin-core fiber [11], sapphire optical fiber [12], micro-fiber [13], and D-shaped fiber [14]. The measurement principle of the sensors depends on the devices, such as the fiber Bragg grating (FBG) [15], long period grating [16], tapered fiber [17], Mach-Zehnder interferometer (MZI) [18], Fabry-Perot interferometer (FPI) [19], and Sagnac loop interferometer [20]. To address practical demands, many researchers have investigated optical fiber sensors to realize multi-parameter measurement. Hu et al. [21] presented a cascaded multi-mode fiber and FBG structure in fiber to

Received: 22 October 2020 / Revised: 23 November 2020

(C) The Author(s) 2020. This article is published with open access at Springerlink.com

DOI: $10.1007 / \mathrm{s} 13320-020-0612-3$

Article type: Regular 
develop a surface plasmon resonance (SPR) sensor for dual-parameter measurement. Li et al. [22] utilized few-mode fiber to fabricate an optical fiber distributed sensor, by analyzing the Brillouin frequency shift in each mode to measure and discriminate temperature and strain. A micro-tip FP cavity, fabricated by focused ion beam milling, was used to measure the temperature and RI in inaccessible locations [23]. Special filter with a dual-wavelength fiber laser was employed to measure RI and temperature by recording the wavelength shift and output power difference of two laser beams [24].

In this work, a probe-shaped optical fiber sensor which can be used for temperature and pressure measurement was proposed and experimentally demonstrated. The sensor is a hybrid structure consisting of an FBG and an FPI with a nano silica diaphragm on the tip, and the total length is less than $2 \mathrm{~mm}$. Herein, the FBG and FPI respond to the environmental temperature and the pressure, respectively. Due to the pressure insensitiveness of the FBG, the cross-sensitivity between temperature and pressure of the FPI can be mostly eliminated by establishing the temperature compensation using the FBG temperature response. The probe-shaped all-silica micro sensor that can achieve dual-parameter measurement has a great potential in particular applications in harsh environments.

\section{Experiments and discussion}

Figure 1(a) shows a structure schematic of a probe-shaped sensor, which consists of an FBG and an FPI with a nano diaphragm. The fabrication process includes two steps. Firstly, a fiber-tip air bubble (FAB) for developing an in-fiber FPI was obtained by the fusion splicing technology which was an improved electrical arc discharge fabrication method to construct an all-silica FPI cavity with an air bubble and a silica nano diaphragm [25]. Secondly, the FBG with grating pitch of $1.089 \mu \mathrm{m}$, corresponding to a second-order Bragg resonance wavelength at $1575 \mathrm{~nm}$, was fabricated by a femtosecond (fs) laser, where a $40 \mathrm{~mW}$ laser was focused into the single mode fiber core by an oil immersed $63 \times$ objective $(\mathrm{NA}=1.4) \quad$ during line-by-line FBG inscription. The corresponding FBG length and FPI cavity length were measured as $\sim 1.7 \mathrm{~mm}$ and $90 \mu \mathrm{m}$, respectively. The reflection spectra of the probe-shaped sensor were recorded by an optical spectrum analyzer (OSA, Yokogawa AQ6370D) with wavelength ranging from $1525 \mathrm{~nm}$ to $1605 \mathrm{~nm}$, as shown in Fig. 1(b). The resonance wavelength of the FBG was $1575.2 \mathrm{~nm}$ and the free spectral range of FP interference was $\sim 13.9 \mathrm{~nm}$ at $1575 \mathrm{~nm}$. The length of FP cavity was calculated as $89.2 \mu \mathrm{m}$.

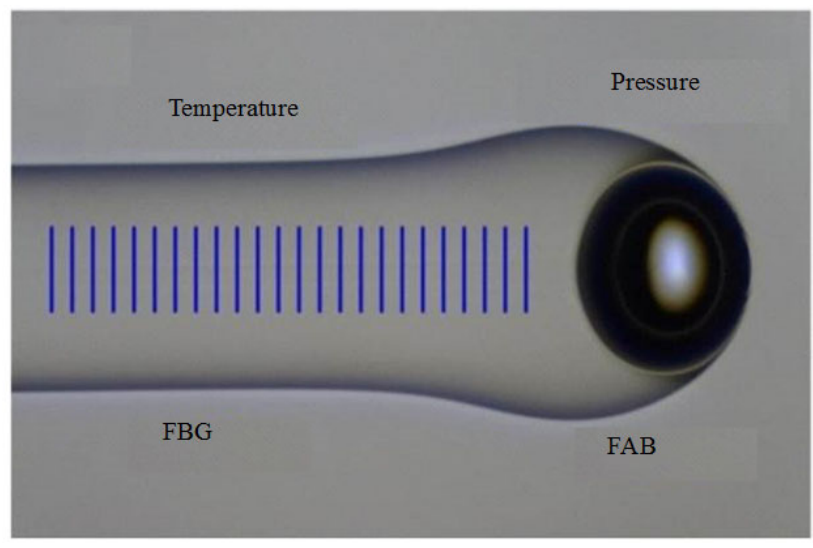

(a)

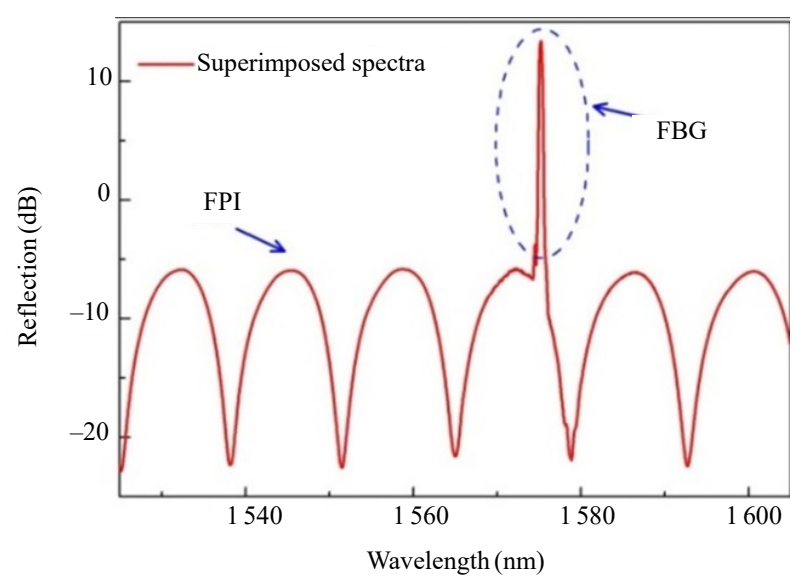

(b)

Fig. 1 Fabricated sample of the probe-shaped sensor: (a) structure schematic of the probe-shaped sensor and (b) the superimposed reflection spectra of the FBG and FPI. 
In the gas pressure measurement, the probe-shaped sensor was sealed in an airtight gas chamber. Chamber pressure was adjusted by coarse and fine valves, and a pressure meter was used to monitor the chamber pressure. The gas pressure response test results are shown in Fig. 2. The evolution of the FPI reflection spectra with the chamber pressure increasing from $0 \mathrm{MPa}$ to $1 \mathrm{MPa}$ and step of 0.1 MPa is shown in Fig.2(b). The FPI dip shifted towards shorter wavelength as the gas

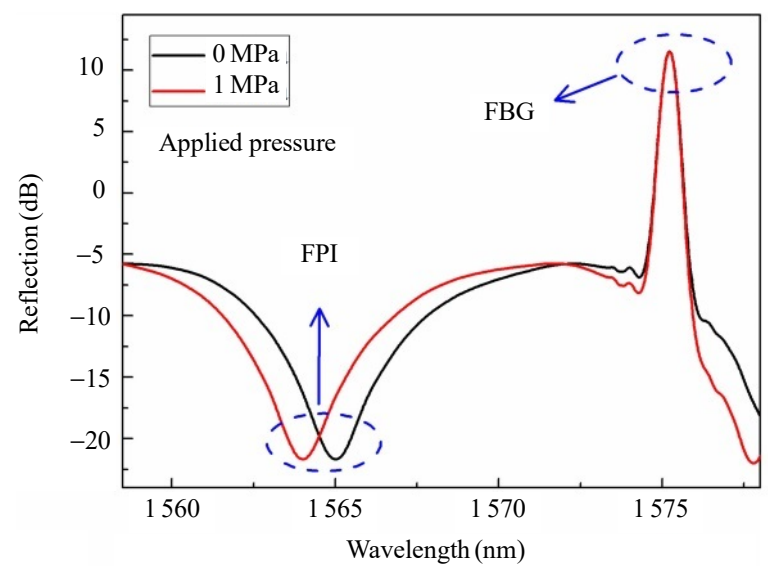

(a)

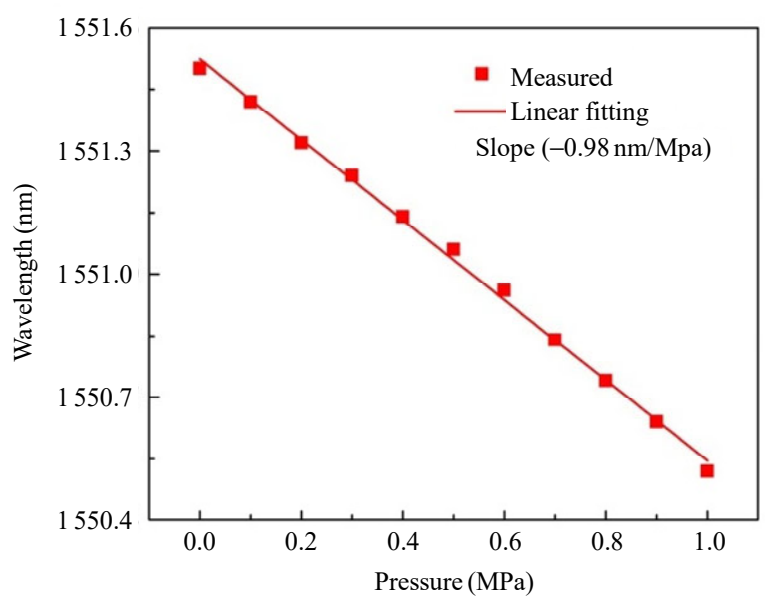

(c) pressure increased, which is because of the deformation of FAB nano diaphragm that leads to a decrease in the cavity length. The linear fitting [Fig. 2(c)] of the pressure test result shows that the pressure sensitivity was $-0.98 \mathrm{~nm} / \mathrm{MPa}$. The Bragg resonance peak had no distinct change in this process. As shown in Fig. 2(d), for the FBG, the change of pressure has negligible effect on the effective RI and the grating period, so the Bragg wavelength remains unchanged.

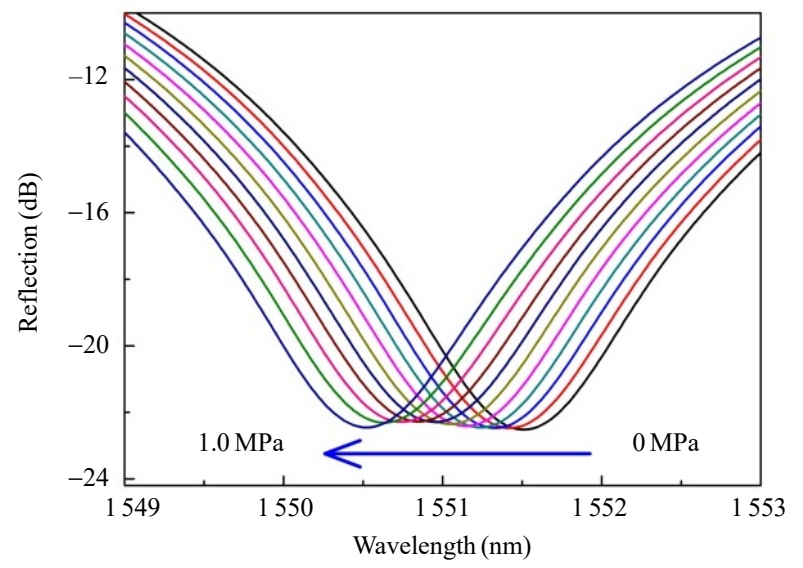

(b)

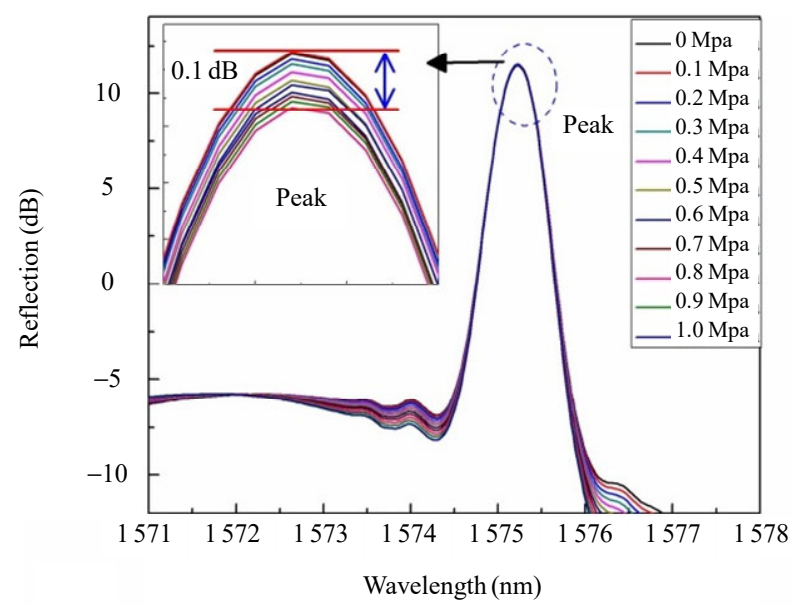

(d)

Fig. 2 Gas pressure response of the probe-shaped sensor: (a) variation of the FPI dip and the FBG peak under the gas pressure at $0 \mathrm{MPa}$ and $1 \mathrm{MPa}$, (b) an enlarged view of spectral evolution of FPI dip for different gas pressures, (c) linear relationship of FPI dip shift and gas pressure, with a slope of $-0.98 \mathrm{~nm} / \mathrm{MPa}$, and (d) the FBG resonance peak evolution under different pressures.

Furthermore, to investigate the temperature response characteristics, the sensor was placed in a temperature control oven. The reflection spectrum was monitored as the temperature increased from $20^{\circ} \mathrm{C}$ to $100^{\circ} \mathrm{C}$ with step of $10^{\circ} \mathrm{C}$. The spectra were recorded after 10 minutes of stabilizing at each temperature step. From Fig. 3(a), it can be observed that as the external temperature rises, the FBG peak 
shifts towards longer wavelength while the FPI dip has no obvious change. The evolution spectra of the FBG temperature response are shown in Fig. 3(b) while its linear fitting is shown in Fig. 3(c). The corresponding temperature sensitivity was $11.1 \mathrm{pm} /{ }^{\circ} \mathrm{C}$ which was similar to the result reported previously [26], and this parameter was mainly affected by the photo-thermal effect. The FPI dip shift shown in Fig. 3(d) is $\sim 120 \mathrm{pm}$ with a temperature sensitivity of $1.4 \mathrm{pm} /{ }^{\circ} \mathrm{C}$. The low

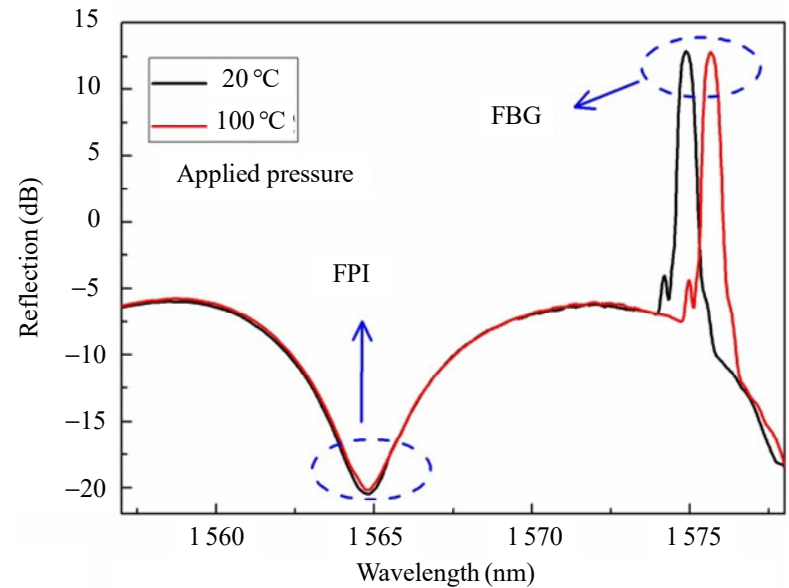

(a)

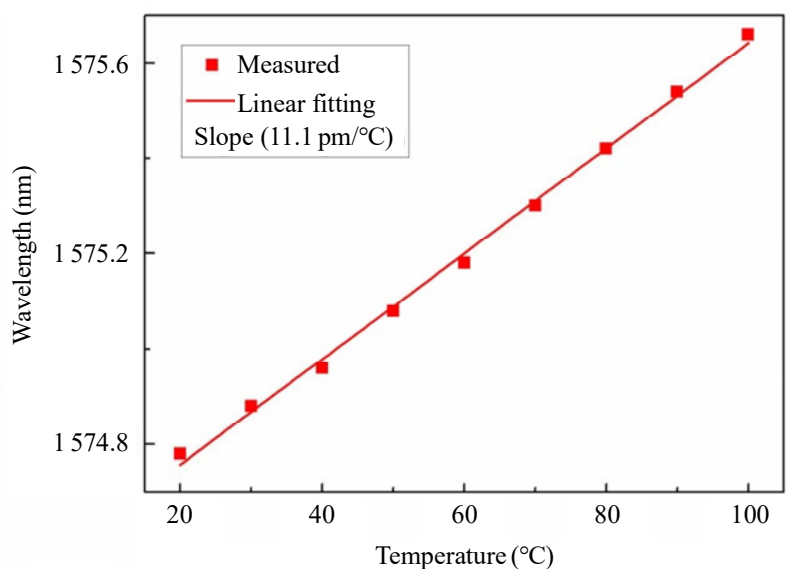

(c) temperature sensitivity of FPI dip can be attributed to that an air cavity structure like FAB is insensitive to external temperature change.

The temperature-induced error of the pressure measurement without temperature compensation was $-1.4286 \mathrm{kPa} /{ }^{\circ} \mathrm{C}$ while the pressure-induced error of the temperature measurement was $\sim 0{ }^{\circ} \mathrm{C} / \mathrm{MPa}$. It can be seen that the probe-shaped sensor has low cross-sensitivity in simultaneous measurement of temperature and pressure.

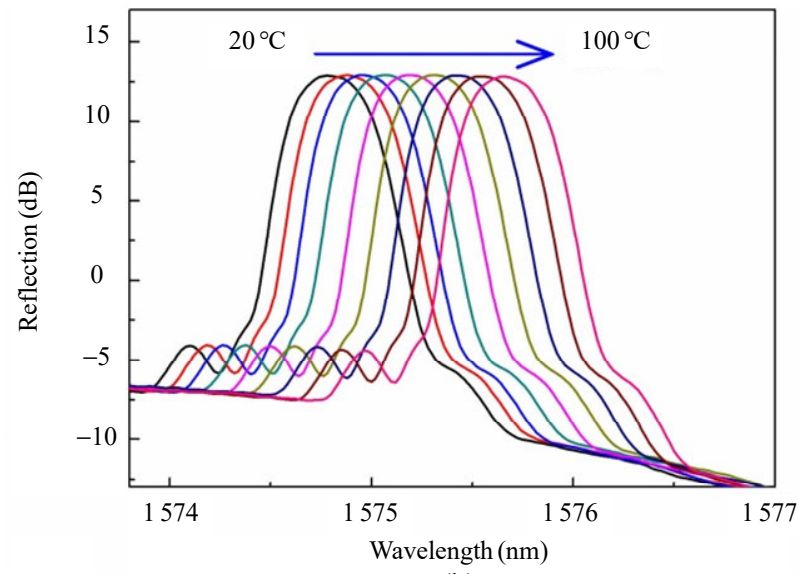

(b)

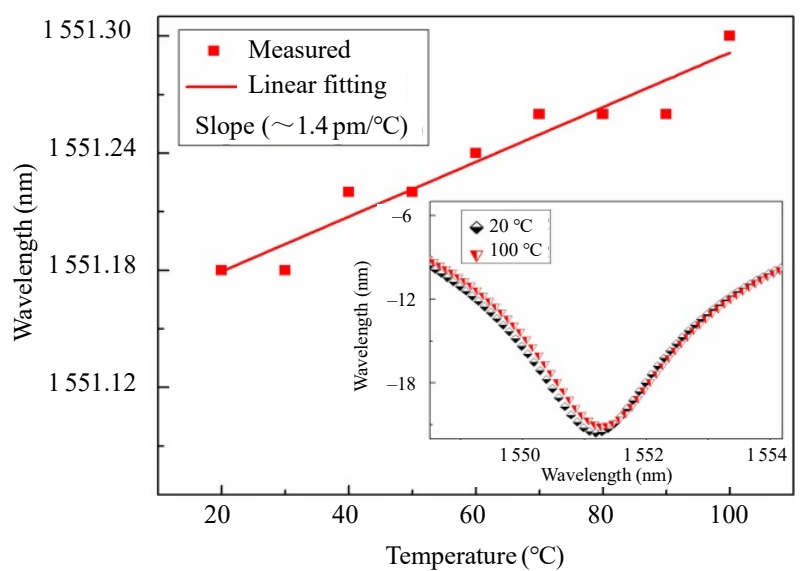

(d)

Fig. 3 Temperature response of the probe-shaped sensor: (a) variation of the FBG peaks and FPI dips at $20{ }^{\circ} \mathrm{C}$ and $100{ }^{\circ} \mathrm{C}$, (b) wavelength shift of FBG peak with the temperature varying from $10{ }^{\circ} \mathrm{C}$ to $100{ }^{\circ} \mathrm{C}$, (c) the linear fitting of Bragg peak wavelength shift with a slope of $\sim 11.10 \mathrm{pm} /{ }^{\circ} \mathrm{C}$, and (d) wavelength shift of FPI dip with the temperature varying from $10{ }^{\circ} \mathrm{C}$ to $100{ }^{\circ} \mathrm{C}$, and the linear fitting slope is $\sim 1.4 \mathrm{pm} /{ }^{\circ} \mathrm{C}$.

To investigate the deformation of the probeshaped sensor under a particular gas pressure, a simulation was performed using commercial finite element analysis software. The simulation employed standard parameters of silica, i.e., silica density of
$2700 \mathrm{~kg} / \mathrm{m}^{3}$, Young's modulus of $73 \mathrm{GPa}$, coefficient of thermal expansion of $5.5 \times 10^{-7}$, and Poisson's ratio of 0.17 . The morphology of the simulation model was extracted from the microscopic image shown in Fig. 1(a). Figure 4(a) illustrates the 
two-dimensional (2D) and three-dimensional (3D) deformation contours of the sensor, which was modeled under the gas pressure of $1 \mathrm{MPa}$. Notably, the modeling results show that when the sensor was subjected to $1 \mathrm{MPa}$ pressure, only the thin diaphragm on the tip of the sensor was deformed while FBG was not affected by pressure, which contributed to the ultra-low, almost zero pressure-induced cross-sensitivity in temperature sensing. The influence of temperature was theoretically investigated by modeling the axial elongation of the sensor at $100^{\circ} \mathrm{C}$. The simulation result is shown in Fig. 4(b). The whole sensor, including FBG and FAB, has inevitable axial elongation due to thermal expansion. The thermal expansion of silica is dominant in such structure, and the elongation of the bubble is far less than that of FBG due to less silica content of the bubble. Therefore, the sensor has low temperature-induced cross-sensitivity in gas pressure sensing.

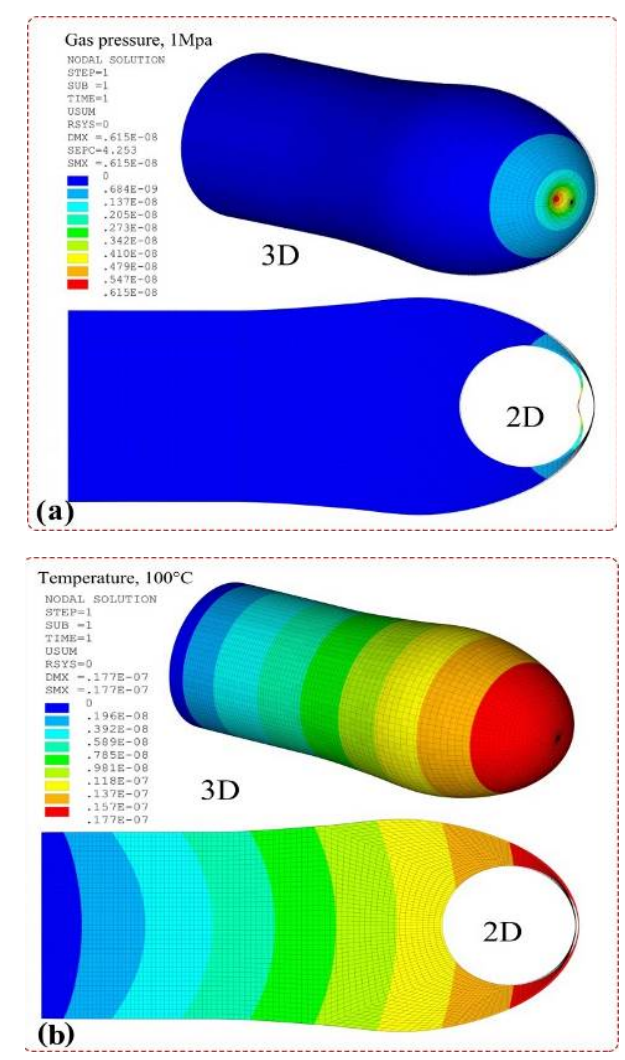

Fig. $43 \mathrm{D}$ and 2D simulated sensor model of (a) the deformation under $1 \mathrm{MPa}$ air pressure and (b) the axial elongation in $100^{\circ} \mathrm{C}$.
To eliminate the cross-sensitivity, temperature compensation is proposed as follows.

The wavelength shifts of FPI dip $\Delta \lambda_{\mathrm{FPI}}$ and Bragg resonance peak $\Delta \lambda_{\mathrm{FBG}}$ against temperature variation $\Delta T$ and pressure variation $\Delta P$ can be described as

$$
\begin{gathered}
\Delta \lambda_{\mathrm{FPI}}=S_{\mathrm{FPI}}^{T} \cdot \Delta T+S_{\mathrm{FPI}}^{P} \cdot \Delta P \\
\Delta \lambda_{\mathrm{FBG}}=S_{\mathrm{FBG}}^{T} \cdot \Delta T+S_{\mathrm{FBG}}^{P} \cdot \Delta P .
\end{gathered}
$$

They can be converted to a matrix form as

$$
\left[\begin{array}{c}
\Delta \boldsymbol{\lambda}_{\mathrm{FPI}} \\
\Delta \boldsymbol{\lambda}_{\mathrm{FBG}}
\end{array}\right]=\left[\begin{array}{cc}
\mathbf{S}_{\mathrm{FPI}}^{T} & \mathbf{S}_{\mathrm{FPI}}^{P} \\
\mathbf{S}_{\mathrm{FBG}}^{T} & \mathbf{S}_{\mathrm{FBG}}^{P}
\end{array}\right]\left[\begin{array}{c}
\boldsymbol{\Delta} \mathbf{T} \\
\boldsymbol{\Delta} \mathbf{P}
\end{array}\right]
$$

where $S_{\mathrm{FPI}}^{T}, S_{\mathrm{FBG}}^{P}, S_{\mathrm{FBG}}^{T}$, and $S_{\mathrm{FPI}}^{P}$ are the temperature sensitivity of FPI, the pressure sensitivity of FPI, the temperature sensitivity of FBG, and the pressure sensitivity of FBG, respectively. The variation of pressure and temperature can be obtained by matrix operation:

$$
\begin{aligned}
{\left[\begin{array}{c}
\boldsymbol{\Delta} \mathbf{T} \\
\boldsymbol{\Delta} \mathbf{P}
\end{array}\right]=} & \frac{1}{S_{\mathrm{FPI}}^{T} \cdot S_{\mathrm{FBG}}^{P}-S_{\mathrm{FBG}}^{T} \cdot S_{\mathrm{FPI}}^{P}} \times \\
& {\left[\begin{array}{cc}
\mathbf{S}_{\mathrm{FBG}}^{P} & -\mathbf{S}_{\mathrm{FPI}}^{P} \\
-\mathbf{S}_{\mathrm{FBG}}^{T} & \mathbf{S}_{\mathrm{FPI}}^{T}
\end{array}\right]\left[\begin{array}{c}
\boldsymbol{\Delta} \boldsymbol{\lambda}_{\mathrm{FPI}} \\
\boldsymbol{\Delta} \boldsymbol{\lambda}_{\mathrm{FBG}}
\end{array}\right] . }
\end{aligned}
$$

The pressure and temperature sensitivities of FPI and FBG were obtained by experiments. As a result, (4) can be further derived as

$$
\left[\begin{array}{l}
\boldsymbol{\Delta} \mathbf{T} \\
\boldsymbol{\Delta} \mathbf{P}
\end{array}\right]=\left[\begin{array}{cc}
0 & 90.0901 \\
-1.0204 & 0.1287
\end{array}\right]\left[\begin{array}{c}
\boldsymbol{\Delta} \boldsymbol{\lambda}_{\mathrm{FPI}} \\
\boldsymbol{\Delta} \boldsymbol{\lambda}_{\mathrm{FBG}}
\end{array}\right]
$$

where the cross-sensitivity is basically removed.

\section{Conclusions}

In conclusion, an all-fiber hybrid sensor consisting of an FPI with silica diaphragm and an FBG was designed and demonstrated. The FBG was fabricated by the femtosecond laser inscription technique, and the bubble cavity was fabricated by arc discharge technology. The pressure sensitivity was $-0.98 \mathrm{~nm} / \mathrm{MPa}$, and temperature sensitivity was $11.10 \mathrm{~nm} /{ }^{\circ} \mathrm{C}$. This sensor exhibited ultra-low crosssensitivities. The temperature-induced error of the pressure measurement was $-1.4286 \mathrm{kPa} /{ }^{\circ} \mathrm{C}$ and pressure-induced error of the temperature 
measurement was $\sim 0^{\circ} \mathrm{C} / \mathrm{MPa}$. Such kind of sensor with low cross-sensitivity is potentially suitable for various applications.

\section{Acknowledgment}

This work was supported by National Natural Science Foundation of China (NSFC) (Grant Nos. 61905165 and 61635007), Natural Science Foundation of Guangdong Province (Grant Nos. 2019A050510047, 2019B1515120042, and 2018A030310581), Education Department of Guangdong Province (Grant No. 2018KQNCX219), and Science and Technology Innovation Commission of Shenzhen (Grant Nos. JCYJ20170412105604705, JCYJ20180305125352956, JCYJ20170818143853289, and JCYJ20170818093743767).

Open Access This article is distributed under the terms of the Creative Commons Attribution 4.0 International License (http://creativecommons.org/licenses/by/4.0/), which permits unrestricted use, distribution, and reproduction in any medium, provided you give appropriate credit to the original author(s) and the source, provide a link to the Creative Commons license, and indicate if changes were made.

\section{References}

[1] L. Nguyen, S. Warren-Smith, H. EbendorffHeidepriem, and T. M.Monro, "Interferometric high temperature sensor using suspended-core optical fibers," Optics Express, 2016, 24(8): 8967-8977.

[2] Y. Yang, S. Saurabh, J. Ward, and S. Chormaic, "High-Q, ultrathin-walled microbubble resonator for aerostatic pressure sensing," Optics Express, 2016, 24(1): 294-299.

[3] L. Niu, C. Zhao, H. Gong, and S. Jin, "Curvature sensor based on two cascading abrupt-tapers modal interferometer in single mode fiber," Optics Communications, 2014, 333: 11-15.

[4] M. Xiong, H. Gong, Z. Wang, C. Zhao, and X. Dong, "Fiber curvature sensor based on spherical-shape structures and long-period grating," Optics and Lasers in Engineering, 2016, 86: 356-359.

[5] G. Woyessa, K. Nielsen, A. Stefani, C. Markos, and O. Bang, "Temperature insensitive hysteresis free highly sensitive polymer optical fiber Bragg grating humidity sensor," Optics Express, 2016, 24(2): 1206-1213.

[6] E. Klantsataya, A. Francois, H. EbendorffHeidepriem, P. Hoffman, and T. M. Monro, "Surface plasmon scattering in exposed core optical fiber for enhanced resolution refractive index sensing," Sensors, 2015, 15(10): 25090-25102.

[7] Q. Liu, S. Li, and H. Chen, "Enhanced sensitivity of temperature sensor by a PCF with a defect core based on Sagnac interferometer," Sensors and Actuators B: Chemical, 2018, 254: 636-641.

[8] Z. Xu, J. Lim, D. Hu, Q. Sun, R. Wong, K. Li, et al., "Investigation of temperature sensing characteristics in selectively infiltrated photonic crystal fiber," Optics Express, 2016, 24(2): 1699-1707.

[9] L. Shao, J. Hu, H. Lu, J. Du, T. Wu, and Y. Wang, "High-sensitivity temperature sensor based on polarization maintaining fiber Sagnac loop," Photonic Sensors, 2019, 9(1): 25-32.

[10] R. Xing, C. Dong, Z. Wang, Y. Wu, Y. Yang, and S. Jian, "Simultaneous strain and temperature sensor based on polarization maintaining fiber and multimode fiber," Optics \& Laser Technology, 2018, 102: 17-21.

[11] Y. Wu, L. Pei, W. Jin, Y. Jiang, Y. Yang, Y. Shen, et al., "Highly sensitive curvature sensor based on asymmetrical twin core fiber and multimode fiber," Optics \& Laser Technology, 2017, 92: 74-79.

[12] X. Xu, J. He, C. Liao, K. Yang, K. Guo, C. Li, et al., "Sapphire fiber Bragg gratings inscribed with a femtosecond laser line-by-line scanning technique," Optics Letters, 2018, 43(19): 4562-4565.

[13] Z. Xu, Y. Luo, Q. Sun, Y. Xiang, P. Shun, and D. Liu, "Switchable single-longitudinal-mode fiber laser based on theta-shaped microfiber filter," IEEE Photonics Technology Letters, 2018, 30(5): 479-482.

[14] Y. Dong, S. Xiao, H. Xiao, J. Liu, C. Sun, and S. Jian, "An optical liquid-level sensor based on D-shape fiber modal interferometer," IEEE Photonics Technology Letters, 2017, 29(13): 1067-1070.

[15] L. Bundalo, K. Nielsen, G. Woyessa, and O. Bang, "Long-term strain response of polymer optical fiber FBG sensors," Optical Materials Express, 2017, 7(3): 967-976.

[16] M. Janczuk-Richter, M. Dominik, E. Razniecka, M. Koba, P. Mikulic, W. J. Bock, et al., "Long-period fiber grating sensor for detection of viruses," Sensors and Actuators B: Chemical, 2017, 250: 32-38.

[17] Y. Tian, W. Wang, N. Wu, X. Zou, and X. Wang, "Tapered optical fiber sensor for label-free detection of biomolecules," Sensors, 2011, 11(4): 3780-3790.

[18] W. Talataisong, D. N. Wang, R. Chitaree, C. Liao, and C. Wang, "Fiber in-line Mach-Zehnder interferometer based on an inner air-cavity for 
high-pressure sensing," Optics Letters, 2015, 40(7): 1220-1222.

[19] J. Zhu, M. Wang, L. Chen, X. Ni, and H. Ni, "An optical fiber Fabry-Perot pressure sensor using corrugated diaphragm and angle polished fiber," Optics Fiber Technology, 2017, 34: 42-46.

[20] L. Shao, Y. Luo, Z. Zhang, X. Zou, B. Luo, W. Pan, et al., "Sensitivity-enhanced temperature sensor with cascaded fiber optic Sagnac interferometers based on Vernier-effect," Optics Communications, 2015, 336: 73-76.

[21] T. Hu, Y. Zhao, and A. Song, "Fiber optic SPR sensor for refractive index and temperature measurement based on MMF-FBG-MMF structure," Sensors and Actuators B: Chemical, 2016, 237: 521-525.

[22] A. Li, Y. Wang, J. Fang, M. Li, B. Kim, and W. Shieh, "Few-mode fiber multi-parameter sensor with distributed temperature and strain discrimination," Optics Letters, 2015, 40(7): 1488-1491.
[23] R. M. André, S. C. Warren-Smith, M. Becker, J. Dellith, M. Rothhardt, M. I. Zibaii, et al., "Simultaneous measurement of temperature and refractive index using focused ion beam milled Fabry-Perot cavities in optical fiber micro-tips," Optics Express, 2016, 24(13): 14053-14065.

[24] B. Yin, S. Wu, M. Wang, W. Liu, H. Li, B. Wu, et al., "High-sensitivity refractive index and temperature sensor based on cascaded dual- wavelength fiber laser and SNHNS interferometer," Optics Express, 2019, 27(1): 252-264.

[25] C. Liao, S. Liu, L. Xu, C. Wang, Y. Wang, Z. Li, et al., "Sub-micron silica diaphragm-based fiber-tip Fabry-Perot interferometer for pressure measurement," Optics Letters, 2014, 39(10): 2827-2830.

[26] L. Luo, S. Liu, Y. Zhao, Y. Chen, K. Yang, K. Guo, et al., "Phase-shifted fiber Bragg grating modulated by a hollow cavity for measuring gas pressure," Optics Letters, 2020, 45(2): 507-510. 